\title{
Sozialstaat unter Druck: \\ auf dem Weg zur Zweiklassenmedizin?
}

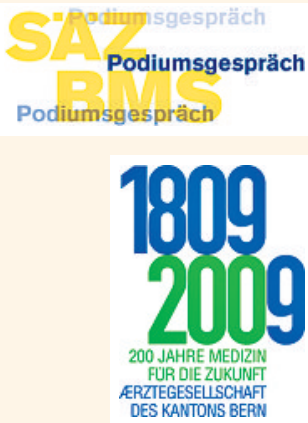

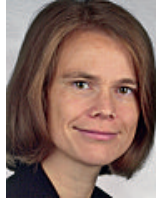

N. Biller-Andorno

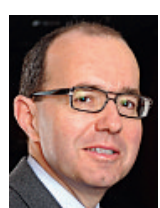

T. B. Cueni

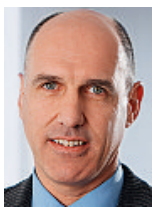

J. de Haller

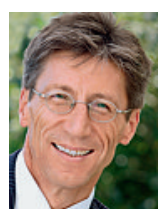

T. Heiniger

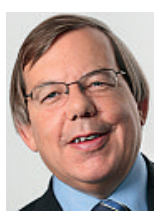

M. Manser

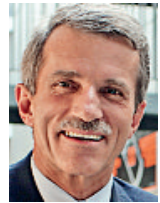

T. Zeltner

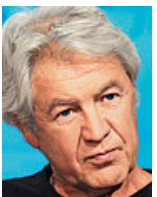

U. Heiniger
Wie positionieren sich die Ärzteschaft und weitere Akteure des Gesundheitswesens? Der Druck auf den Sozialstaat hat in den letzten Jahren zugenommen: Nicht erst seit Finanzkrise und Rezession prägen ökonomische Aspekte und Sparmassnahmen die Debatten auch im Gesundheitswesen und engen den Handlungsspielraum der Akteure auf allen Ebenen zunehmend ein. In verschiedenen Bereichen der Medizin stehen tiefgreifende Veränderungen an, so im Spitalsektor mit der Einführung der DRGs (Diagnosis Related Groups oder Fallpauschalen) oder im ambulanten Sektor mit dem teilweise bereits manifesten Mangel an Grundversorgern.

\section{Unterschiedliche Rezepte}

Die Ideen und Vorschläge, wie diesen Entwicklungen zu begegnen ist, gehen weit auseinander. Welche Gesundheitsversorgung können und wollen wir uns in Zukunft als Gesellschaft noch leisten? Hinter unterschiedlichen politischen Auffassungen und Lösungsvorschlägen stehen unterschiedliche Menschen- und Gesellschaftsbilder. In Stichworten wie «Eigenverantwortung», «Solidarität im Gesundheitswesen», «Zweiklassenmedizin» oder «Rationierung», die interessenpolitisch mitunter gezielt eingesetzt werden, kristallisieren sich einige der aktuellen Problemzonen.

\section{Diskutieren Sie mit}

Die Ärzteschaft ist von diesen Veränderungen vital betroffen und gefordert, sich als Berufsgruppe im Zusammenspiel der unterschiedlichen Kräfte im Gesundheitswesen einzubringen, Position zu beziehen und die Standesinteressen wirkungsvoll zu vertreten. Eine Podiumsdiskussion der Schweizerischen Ärztezeitung mit Meinungsführern aus Ärzteschaft, Politik, Ethik, Verwaltung, Krankenkassen und Wirtschaft soll die Problemzonen des «Patienten Sozialstaat» aus Sicht der verschiedenen Akteure im Gesundheitswesen analysieren und die jeweiligen Positionen herausarbeiten. In der anschliessenden offenen Diskussion stellen sich die Teilnehmer den Fragen des Publikums. Auf diese
Weise soll ein interessanter und konstruktiver Beitrag zur Debatte aktueller Fragen des Gesundheitswesens geleistet werden.

Es diskutieren unter der Leitung von Dr. phil. Ueli Heiniger (freischaffender Moderator, langjähriger Leiter der Diskussionssendung «Der Club» des Schweizer Fernsehens DRS):

- Prof. Dr. med. et phil. Nikola Biller-Andorno, Direktorin des Instituts für Biomedizinische Ethik der Universität Zürich;

- Thomas B. Cueni, Generalsekretär Interpharma; - Dr. med. Jacques de Haller, Präsident FMH;

- Dr. iur. Thomas Heiniger, Gesundheitsdirektor Kanton Zürich;

- Manfred Manser, Vorsitzender der Konzernleitung der Helsana Krankenversicherung;

- Prof. Dr. med. Thomas Zeltner, Direktor BAG.

\section{Eintritt frei - Anmeldung erforderlich}

Eine Anmeldung für die Podiumsdiskussion mit anschliessendem Apéro ist trotz freiem Eintritt zwingend erforderlich, da die Teilnehmerzahl begrenzt ist. Sie kann bis Freitag, 16. Juni 2009, via E-Mail an redaktion.saez@emh.ch oder via Fax an 0614678556 erfolgen. Bitte Ihren Namen und das Stichwort «Anmeldung zum SÄZ-Podium vom 24. Juni» angeben. Auch telefonische Anmeldungen sind unter 0614678572 möglich.

Die Veranstaltung wird in Zusammenarbeit mit der Ärztegesellschaft des Kantons Bern organisiert, die in diesem Jahr ihr zweihundertjähriges Bestehen feiert.

Die Durchführung des Anlasses wird möglich dank grosszügiger Unterstützung durch Interpharma, den Verband der forschenden pharmazeutischen Industrie. Die Verantwortung für Konzept und Inhalt des Podiums liegt ausschliesslich bei der Schweizerischen Ärztezeitung. 